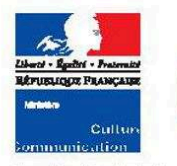

Secrétariat général

Service de la

politiques culturelles

et de l'innovation

Département

des études,

de la prospective
et des statistiques

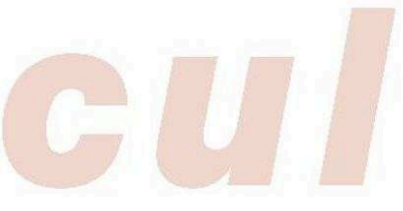

82, rue Saint-Honoré, 75033 Paris cedex 01

중 0140157917 -圂 0140157999

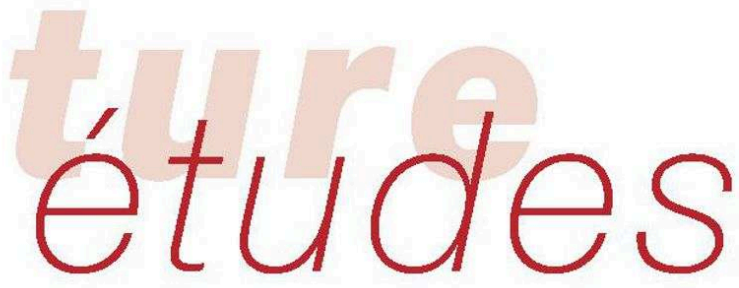

\title{
Les galeries d'art contemporain en France en 2012
}

\section{François Rouet}

\section{Contemporary art galleries in France in 2012}

Les galeries d'art contemporain jouent un rôle essentiel dans l'émergence et la construction de la notoriété des artistes et, partant, la vitalité de la scène artistique française. Dans ce contexte, comprendre les conditions d'exercice de leur activité et apprécier comment pérennité peut rime avec prise de risque s'avère une préoccupation centrale pour la politique en faveur des arts plastiques que mène le ministère de la Culture et de la Communication'

Toutes les enquêtes menées sur les galeries d'art affrontent une double question : d'abord celle du champ, en l'absence d'une activité référencée dans la nomenclature d'activités française (NAF rév. 2) pour la galerie, et a fortiori pour la galerie d'art contemporain, ce qui oblige à croiser les sources et fichiers à la fois accessibles et pertinents; ensuite, celle de savoir comment distinguer les galeries qualifiées « de promotion» qui s'attachent plus particulièrement à la recherche de nouveaux artistes. En effet, le marché de l'ar contemporain s'organise autour de ce qui a été dénommé la «convention d'originalité » suivant laquelle la valeur des ceuvres dépend de leur caractère novateur. Cela le distingue d'une production et d'un marché artistiques qui, pour être également contemporains, relèvent plus d'une convention que l'on pourrait qualifier d'《artisanale », s'attachant à la bonne facture, aux qualités émotionnelles et décoratives, au rendu de l'éventuel sujet. À cet égard, les solutions retenues sont diverses et parfois peu satisfaisantes: distinguer les galeries spécialisées dans l'art figuratif des autres (enquête de 1999), se limiter à un ensemble de galeries considérées a priori comme « de promotion» (2005) ou aux diffuseurs d'artistes vivants inscrits à la Maison des artistes (2009).

La présente enquête a choisi une autre méthode : d'abord repérer le plus largement possible à partir des différentes sources disponibles, comme cela était fait simultanément pour les autres commerces de l'art, les unités qualifiées de galeries d'art potentiellement actives sur le premier marché des cuvres sortant d'atelier; ensuite, introduire dans son questionnaire des questions permettant a priori de cerner le travail de promotion au travers de différentes caractérisations possibles: financement de la production d'cuvres, organisation de premières expositions d'artistes... En tout état de cause, la population enquêtée excède donc largement les seules galeries de promotion pour inclure des galeries promouvant des œuvres ne nécessitant guère de certification de la valeur (le genre classique ou moderne au sens de Nathalie Heinich, par opposition au genre contemporain ${ }^{2}$ ).

Ainsi, une acception initiale large et l'hétérogénéite structurelle des galeries sur le plan artistique doivent être prises en compte dans l' interprétation des résultats, en particulier pour l'ampleur de la population des galeries, leurs caractéristiques et leur importance globale en termes de chiffre d'affaires et d'emploi. D'autant que cette hétérogénéité en recoupe une autre quant à la taille et à l'implanta-

\footnotetext{
* Enquête réalisée par Bluestone sous la direction de Pierre Capelle et Myriam Marcetteau pour le DEPS.

1. Plusieurs enquêtes antérieures avaient porté sur les galeries d'art contemporain : étude, à la demande du DEPS, de Françoise Benhamou, Nathalie Moureau et Dominique Sagot-Duvauroux ayant enquêté les galeries d'art contemporain parisiennes en 1999, voir F. BENHAMOU, N. MOUREAU, D. SAGOTDuvauroux, les Galeries d'art contemporain en France. Portrait et enjeux dans un marché mondialisé, Paris, Ministère de la Culture et de la Communication, DEPS/La Documentation française, coll. "Questions de culture », 2001 ; puis enquête menée par CSA à la demande du Comité professionnel des galeries d'art en 2005 et enfin exploitation des données de la Maison des artistes réalisée sur la période 2005-2008 dans le cadre d'une investigation plus large sur la diffusion et la valorisation de l'art actuel en région à la demande du DEPS, voir Muriel de VRIÈsE, Bénédicte MARTIN, Corinne MELIN, Nathalie MoUREAU, Dominique SAGOT-Duvauroux, Diffusion et valorisation de l'art actuel en région. Une étude des agglomération du Havre, de Lyon, de Montpellier, Nantes et Rouen, Paris, Ministère de la Culture et de la Communication, DEPS, coll. «Culture études », 2011-1.
} 2. Nathalie HejN]CH, le Triple Jeu de l'art contemporain, Paris, Minuit, 1998. 


\section{Les galeries d'art contemporain en France en 2012}

Contemporary art galleries in France in 2012

\section{François Rouet}

Éditeur : Département des études, de la prospective et des statistiques Lieu d'édition : Paris

Année d'édition : 2013

Date de mise en ligne : 8 juillet 2015

Collection : Culture études

ISBN électronique : 9782111399099

\section{Sbooks}

http://books.openedition.org

\section{Édition imprimée}

Date de publication : 1 juin 2013

Nombre de pages : 12

\section{Référence électronique}

ROUET, François. Les galeries d'art contemporain en France en 2012. Nouvelle édition [en ligne]. Paris

Département des études, de la prospective et des statistiques, 2013 (généré le 25 avril 2021).

Disponible sur Internet : <http://books.openedition.org/deps/119>. ISBN : 9782111399099.

(C) Département des études, de la prospective et des statistiques, 2013

Creative Commons - Attribution - Pas d'Utilisation Commerciale 3.0 non transposé - CC BY-NC 3.0 

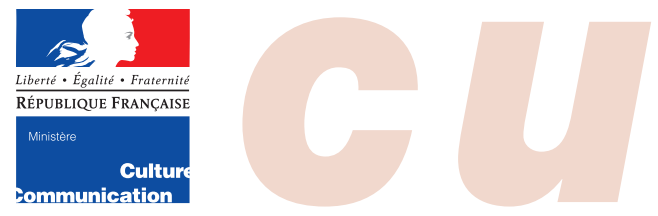

Secrétariat général

Service de la

coordination des

politiques culturelles

et de l'innovation

Département

des études,

de la prospective

et des statistiques

\section{Les galeries d'art contemporain en France en 2012}

\section{François Rouet*}

\section{Contemporary art galleries in France in 2012}

Les galeries d'art contemporain jouent un rôle essentiel dans l'émergence et la construction de la notoriété des artistes et, partant, la vitalité de la scène artistique française. Dans ce contexte, comprendre les conditions d'exercice de leur activité et apprécier comment pérennité peut rimer avec prise de risque s'avère une préoccupation centrale pour la politique en faveur des arts plastiques que mène le ministère de la Culture et de la Communication ${ }^{1}$.

Toutes les enquêtes menées sur les galeries d'art affrontent une double question : d'abord celle du champ, en l'absence d'une activité référencée dans la nomenclature d'activités française (NAF rév. 2) pour la galerie, et a fortiori pour la galerie d'art contemporain, ce qui oblige à croiser les sources et fichiers à la fois accessibles et pertinents ; ensuite, celle de savoir comment distinguer les galeries qualifiées «de promotion » qui s'attachent plus particulièrement à la recherche de nouveaux artistes. En effet, le marché de l'art contemporain s'organise autour de ce qui a été dénommé la «convention d'originalité » suivant laquelle la valeur des œuvres dépend de leur caractère novateur. Cela le distingue d'une production et d'un marché artistiques qui, pour être également contemporains, relèvent plus d'une convention que l'on pourrait qualifier d' «artisanale», s'attachant à la bonne facture, aux qualités émotionnelles et décoratives, au rendu de l'éventuel sujet. À cet égard, les solutions retenues sont diverses et parfois peu satisfaisantes : distinguer les galeries spécialisées dans l'art figuratif des autres (enquête de 1999), se limiter à un ensemble de galeries considérées a priori comme « de promotion » (2005) ou aux diffuseurs d'artistes vivants inscrits à la Maison des artistes (2009).

La présente enquête a choisi une autre méthode : d'abord repérer le plus largement possible à partir des différentes sources disponibles, comme cela était fait simultanément pour les autres commerces de l'art, les unités qualifiées de galeries d'art potentiellement actives sur le premier marché des œuvres sortant d'atelier; ensuite, introduire dans son questionnaire des questions permettant a priori de cerner le travail de promotion au travers de différentes caractérisations possibles : financement de la production d'œuvres, organisation de premières expositions d'artistes... En tout état de cause, la population enquêtée excède donc largement les seules galeries de promotion pour inclure des galeries promouvant des œuvres ne nécessitant guère de certification de la valeur (le genre classique ou moderne au sens de Nathalie Heinich, par opposition au genre contemporain ${ }^{2}$ ).

Ainsi, une acception initiale large et l'hétérogénéité structurelle des galeries sur le plan artistique doivent être prises en compte dans l'interprétation des résultats, en particulier pour l'ampleur de la population des galeries, leurs caractéristiques et leur importance globale en termes de chiffre d'affaires et d'emploi. D'autant que cette hétérogénéité en recoupe une autre quant à la taille et à l'implanta-

\footnotetext{
* Enquête réalisée par Bluestone sous la direction de Pierre Capelle et Myriam Marcetteau pour le DEPS.

1. Plusieurs enquêtes antérieures avaient porté sur les galeries d'art contemporain : étude, à la demande du DEPS, de Françoise Benhamou, Nathalie Moureau et Dominique Sagot-Duvauroux ayant enquêté les galeries d'art contemporain parisiennes en 1999, voir F. BENHAMOU, N. MouREAU, D. SAGOTDuvauroux, les Galeries d'art contemporain en France. Portrait et enjeux dans un marché mondialisé, Paris, Ministère de la Culture et de la Communication, DEPS/La Documentation française, coll. "Questions de culture », 2001 ; puis enquête menée par CSA à la demande du Comité professionnel des galeries d'art en 2005 et enfin exploitation des données de la Maison des artistes réalisée sur la période 2006-2008 dans le cadre d'une investigation plus large sur la diffusion et la valorisation de l'art actuel en région à la demande du DEPS, voir Muriel de VRIÈSE, Bénédicte MARTIN, Corinne Melin, Nathalie Moureau, Dominique Sagot-Duvauroux, Diffusion et valorisation de l'art actuel en région. Une étude des agglomération du Havre, de Lyon, de Montpellier, Nantes et Rouen, Paris, Ministère de la Culture et de la Communication, DEPS, coll. «Culture études », 2011-1.

2. Nathalie HeINICH, le Triple Jeu de l'art contemporain, Paris, Minuit, 1998.
} 
tion géographique des galeries au sein d'une population où voisinent, comme souvent dans les secteurs culturels, une majorité de très petites structures et un nombre très limité de grandes galeries. La netteté du découpage typologique réalisé n'en est que plus significative.

\section{Population des galeries D'ART CONTEMPORAIN FRANÇAISES EN 2012}

La population constituée est celle des galeries supposées intervenir sur le premier marché de l'art, c'est-à-dire intervenant dans le négoce d'œuvres d'artistes en activité. On parle alors d'œuvres et de galeries d'art contemporain. Selon ce critère et après expertise des différentes sources disponibles (voir Éléments de méthodologie), 2191 galeries d'art contemporain ont été identifiées en France, sachant qu'une partie d'entre elles intervient également sur le second marché de la revente d'œuvres. Cette population qui rassemble tous types de galeries quelles que soient leurs orientations artistiques s'avère sensiblement plus importante que celle des seules galeries cotisant à la Maison des artistes qui sont plus d'un millier, et que celles enquêtées précédemment, au nombre de quelques centaines. Par ailleurs, en 2006, suivant l'enquête Commerce de l'Insee, 1491 entreprises avaient indiqué réaliser une part de leur chiffre d'affaires avec des ventes d'art contemporain et 1170 s'y consacrer presque totalement ${ }^{3}$.

Les galeries d'art contemporain sont très inégalement réparties entres les régions:

- la seule région Île-de-France (y compris Paris) comporte 1151 galeries d'art contemporain, soit $53 \%$ du total ;

- le Sud de la France concentre plus d'un quart du total des galeries d'art françaises. Provence-Alpes-Côte d'Azur est la seconde région française en nombre de galeries actives en matière d'art contemporain avec 244 sociétés (soit $11 \%$ de l'ensemble des galeries françaises), suivie de la région Rhône-Alpes (139 galeries soit $6 \%$ des galeries françaises). Cette densité au sud du pays peut s'expliquer du point de vue de l'offre : ces régions sont le terreau des nombreux courants artistiques ayant émergé au cours des cinq dernières décennies, à l'image de l'École de Nice, du Nouveau Réalisme, du groupe Support-Surface ou encore de la Figuration libre. La diffusion de cette production a été relayée par le développement de nombreuses galeries spécialisées, dont certaines de renommée internationale. La demande touristique pour un art figuratif peut, quant à elle, expliquer la présence de galeries qui ne sont pas « de promotion » dans des régions touristiques.

- au total, cinq régions seulement (Île-de-France, ProvenceAlpes-Côte-d'azur, Bretagne, Rhône-Alpes, Aquitaine) regroupent près de $80 \%$ des galeries d'art contemporain en France. Cette proportion est proche de celle des diffuseurs d'art actuel cotisant à la Maison des artistes sur la

\section{Carte - Répartition géographique des galeries d'art françaises actives sur le premier marché en 2012}

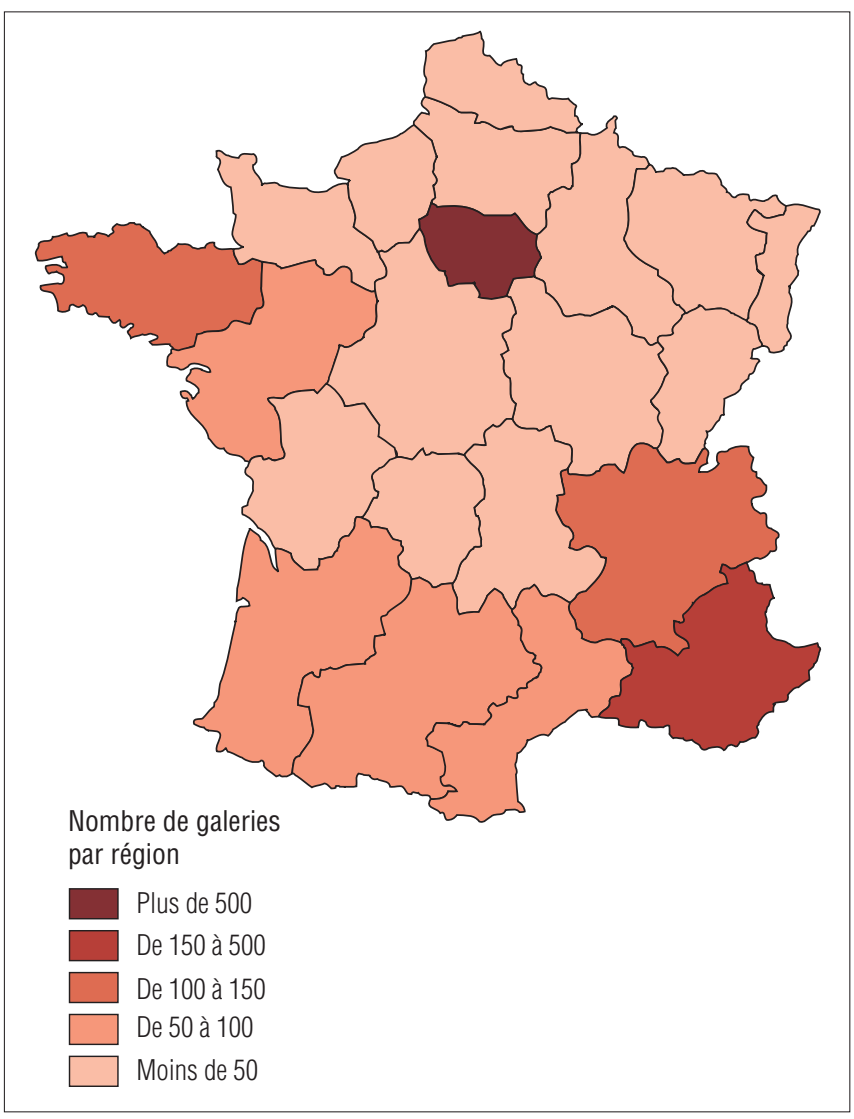

Source : DEPS, Ministère de la Culture et de la Communication, 2013.

période 2006-2008 (73\%) ${ }^{4}$. À l'inverse, les galeries d'art contemporain sont très peu présentes sur près des deux tiers du territoire.

Ces inégalités de répartition géographique sont redoublées par de fortes disparités d'une région à l'autre en termes de chiffre d'affaires des galeries d'art contemporain. Ces fortes disparités entre galeries de régions différentes mais aussi entre galeries d'une même région, qu'elles soient très nombreuses comme à Paris ou très peu nombreuses comme dans la plupart des régions, rendent les valeurs moyennes prises isolément peu significatives.

Paris accueille ainsi plus de 1000 galeries, soit $48 \%$ du total des galeries d'art contemporain repérées en France 5 . Au sein de la capitale, berceau du marché de l'art français, on observe une forte disparité de la répartition des galeries d'art contemporain entre quartiers. Les galeries parisiennes se concentrent principalement dans trois quartiers, les $3^{\mathrm{e}}, 6^{\mathrm{e}}$ et $8^{\mathrm{e}}$ arrondissements, qui accueillent ainsi plus de $52 \%$ des galeries de la capitale mais totalisent, d'après l'enquête, $82 \%$ du chiffre d'affaires total de ces galeries.

- Le $3^{\mathrm{e}}$ arrondissement regroupe 206 galeries. Dans le contexte favorable de l'ouverture du musée national d'Art moderne au sein du centre Pompidou ainsi que d'un fon-

3. François RouET, les Entreprises du commerce du marché de l'art, Paris, Ministère de la Culture et de la Communicaiton, DEPS, coll. « Culture chiffres », avril 2009.

4. Ces diffuseurs sont à $92 \%$ des galeries, aux côtés d'antiquaires, éditeurs...

5. Les galeries franciliennes hors Paris ne sont qu'une centaine (92). 
Graphique 1-Part de la population totale et du chiffre d'affaires déclaré des galeries d'art en 2011

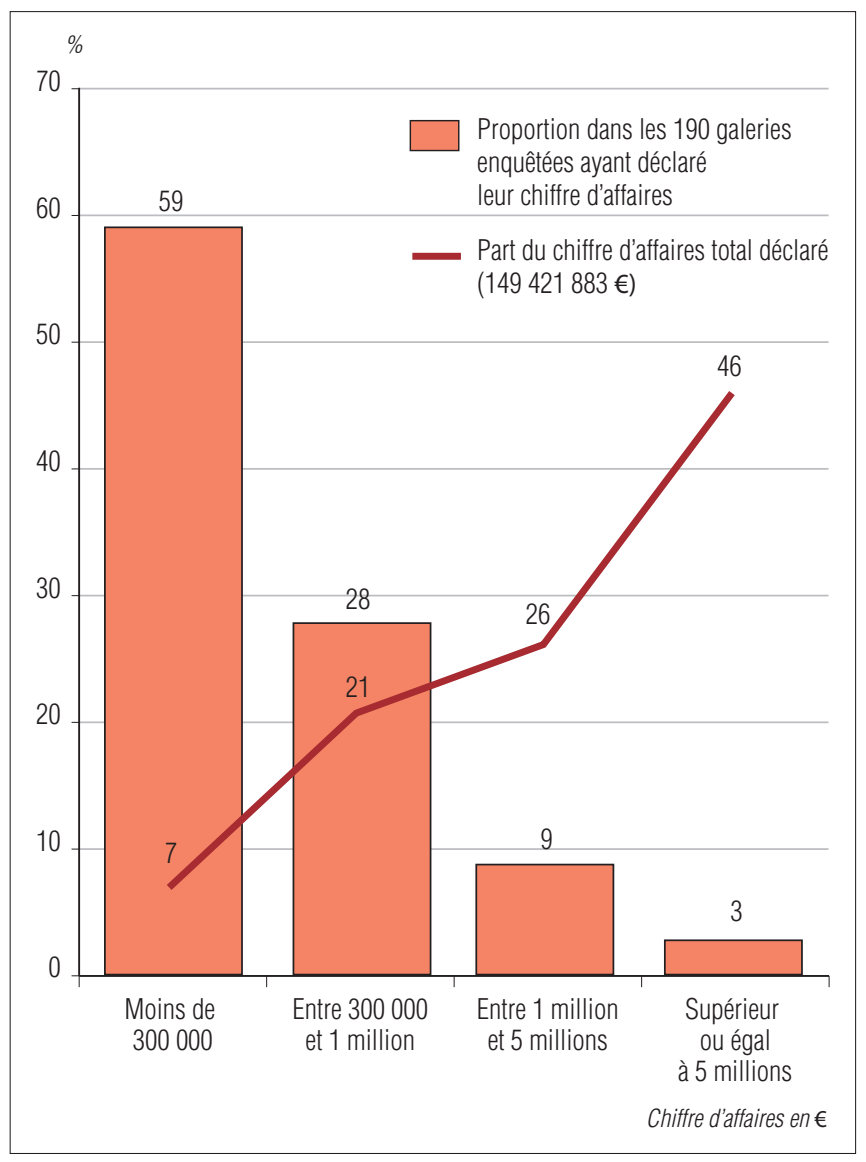

Source : DEPS, Ministère de la Culture et de la Communication, 2013.

cier attractif dans les années 1970-1990, le quartier est devenu un lieu d'implantation de jeunes galeries spécialisées dans l'art contemporain. Le chiffre d'affaires annuel moyen s'élève à 1,15 million d'euros et l'ancienneté moyenne déclarée par les galeries de ce quartier n'est que de 16 ans.

- Le $6^{\mathrm{e}}$ arrondissement compte 189 galeries dans le triangle traditionnel de Saint-Germain-des-Prés formé par les rues Bonaparte, Saint-Sulpice et le quai des Grands-Augustins. Ce quartier, qui abrite l'École nationale supérieure des beaux-arts ainsi que plusieurs rues célèbres pour leurs galeries (rue de Seine, rue des Saints-Pères, rue Guénégaud...), est devenu après-guerre le cœur historique du monde de l'art. L'ancienneté de ses galeries est supérieure à celle de leurs consœurs (23 ans en moyenne) et leur chiffre d'affaires moyen est particulièrement élevé (1,9 millions d'euros).

- Le $8^{\mathrm{e}}$ arrondissement accueille 152 galeries d'art contemporain. Ce quartier est caractérisé par une forte concentration du commerce de luxe, mais aussi par la proximité du Grand Palais, où se tiennent la Fiac et Art Paris, et des salles des ventes des trois premières maisons d'enchères (Christie's, Sotheby's, Artcurial) sur le marché national. Le chiffre d'affaires moyen est de 1,07 million d'euros et l'ancienneté moyenne des galeries de 22 ans.

La concentration des galeries d'art contemporain françaises dans la capitale est encore plus forte en termes de chiffre d'affaires qu'en termes de population. Le chiffre d'affaires moyen des galeries parisiennes atteint 1,15 million d'euros contre tout juste 241000 euros en région. Les galeries parisiennes réalisent ainsi $86 \%$ du montant du chiffre d'affaires national.

Cela est dû en partie au fait que les galeries d'art contemporain françaises les plus importantes en chiffre d'affaires sont implantées à Paris, d'où une forte disparité au sein même des galeries parisiennes (ou franciliennes). Ainsi, parmi les galeries franciliennes, plus de la moitié ont un volume d'activité inférieur à 300000 euros, et un tiers entre 300000 et 1000000 d'euros. En revanche, $5 \%$ des galeries parisiennes réalisent plus de 5 millions d'euros de chiffre d'affaires, un montant que n'atteindrait aucune galerie en région.

Les sources et enquêtes précédentes donnaient des chiffres d'affaires moyens un peu moins élevés : de l'ordre de 300000 à 400000 euros (courants) en 2008, et environ 800000 euros pour les seules galeries parisiennes en 1999, contre respectivement 800000 euros et 1,1 million d'euros en 2012.

Le graphique 1 témoigne d'une concentration significative du chiffre d'affaires. $12 \%$ des galeries réalisent plus d'un million de chiffre d'affaires annuel soit $72 \%$ du chiffre d'affaires total, un résultat voisin du 80/20 habituel dans d'autres secteurs culturels. Cet important écart dans chaque strate de chiffre d'affaires entre part du chiffre d'affaires total et pourcentage de galeries concernées reflète des disparités importantes de taille entre galeries, qui renvoient à des différences significatives dans leur économie, l'orientation de leur activité et leur positionnement stratégique.

\section{Caractéristiques des galeries D'ART CONTEMPORAIN EN FRANCE}

\section{Présence sur le second marché et ancienneté expliquent des volumes d'affaires très inégaux}

Près de trois galeries sur quatre ( $72 \%$ ) travaillent exclusivement sur le premier marché, ne proposant ainsi que des œuvres issues directement des lieux de production. Leur activité est donc totalement dédiée à la promotion des artistes qu'elles représentent. À l'opposé, parmi les galeries proposant des œuvres d'artistes en activité, $28 \%$ déclarent intervenir aussi sur le second marché, c'est-à-dire qu'elles achètent et revendent des biens ayant déjà fait l'objet d'une première transaction.

Les galeries d'art contemporain qui interviennent également sur le second marché réalisent des chiffres d'affaires sensiblement plus élevés que les autres. Le chiffre d'affaires moyen pour les galeries qui interviennent également sur le second marché est ainsi double de celui des galeries exclusivement tournées vers le premier marché en province, et presque quadruple en Île-de-France : on retrouve là le poids des plus grandes galeries parisiennes, très présentes sur le second marché. Globalement, les galeries actives sur le second marché réalisent plus de $60 \%$ du chiffre d'affaires 
Graphique 2 - Chiffre d'affaires des galeries interrogées selon la région et l'intervention sur le premier et le second marché en 2011

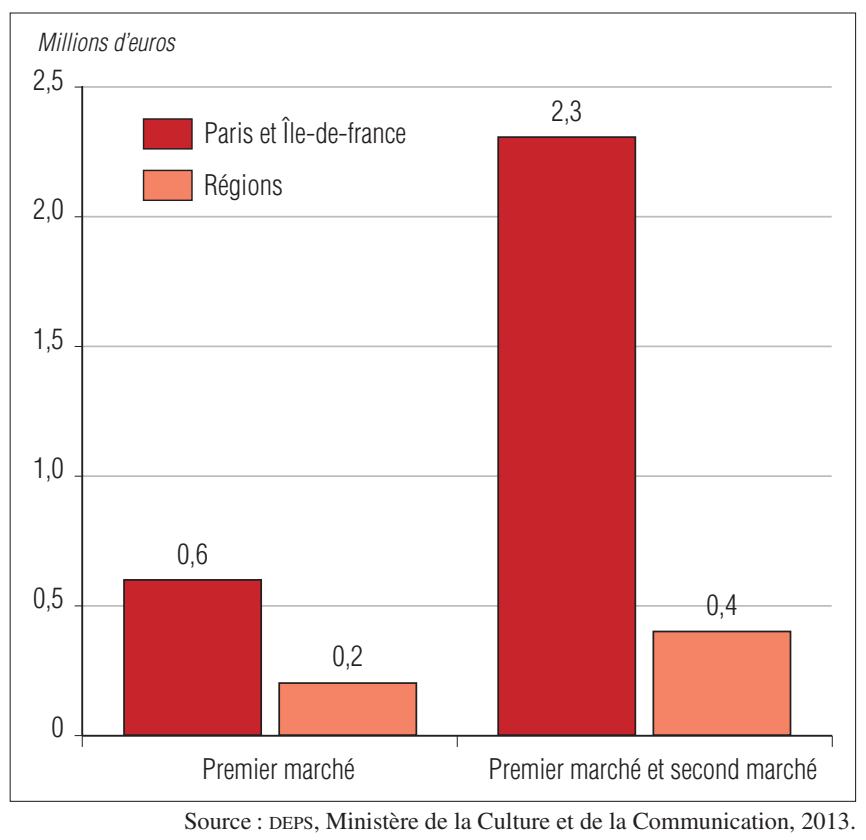

\section{Graphique 3 - Chiffre d'affaires moyen selon la localisation et l'ancienneté des galeries en 2011}

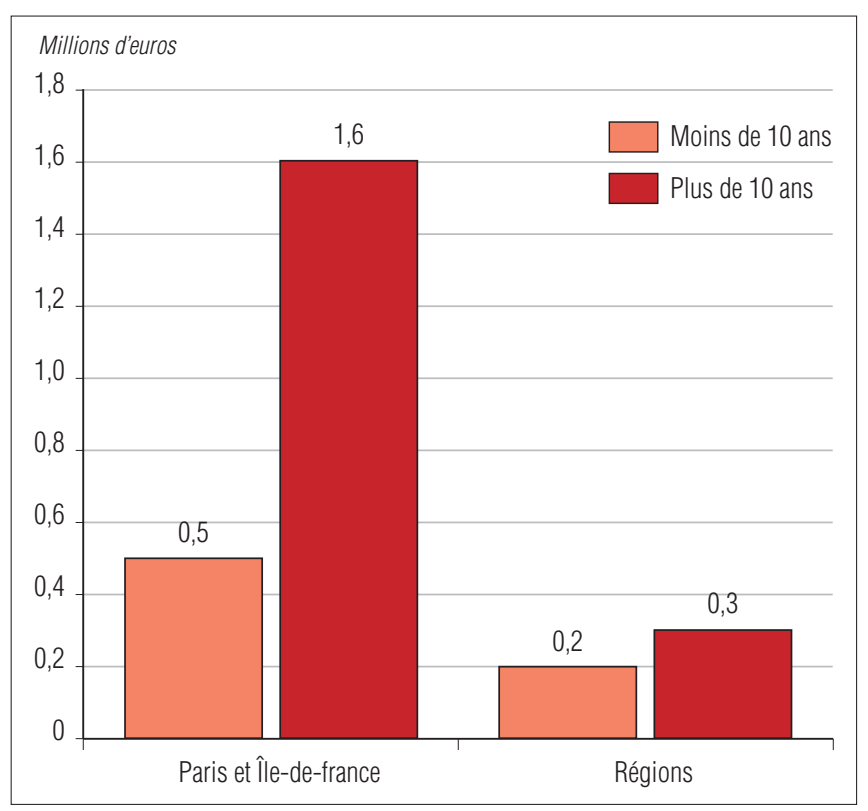

Source : DEPS, Ministère de la Culture et de la Communication, 2013.

total alors qu'elles ne représentent que $28 \%$ des galeries (graphique 2).

S'esquisse ici un choix stratégique important autour de la participation ou non au second marché. Plus générateur de volume d'affaires que le premier, le second marché s'avère par ailleurs indéniablement moins risqué que le premier; s'y positionner pour une galerie d'art contemporain permet de réduire le risque global de son activité, sachant que les potentialités de plus-value y sont plus assurées, quitte à ce qu'elles soient peut-être moins fortes.
Graphique 4-Répartition des galeries enquêtées selon le prix minimum des ouvres d'artistes vivants proposés à la vente en 2011

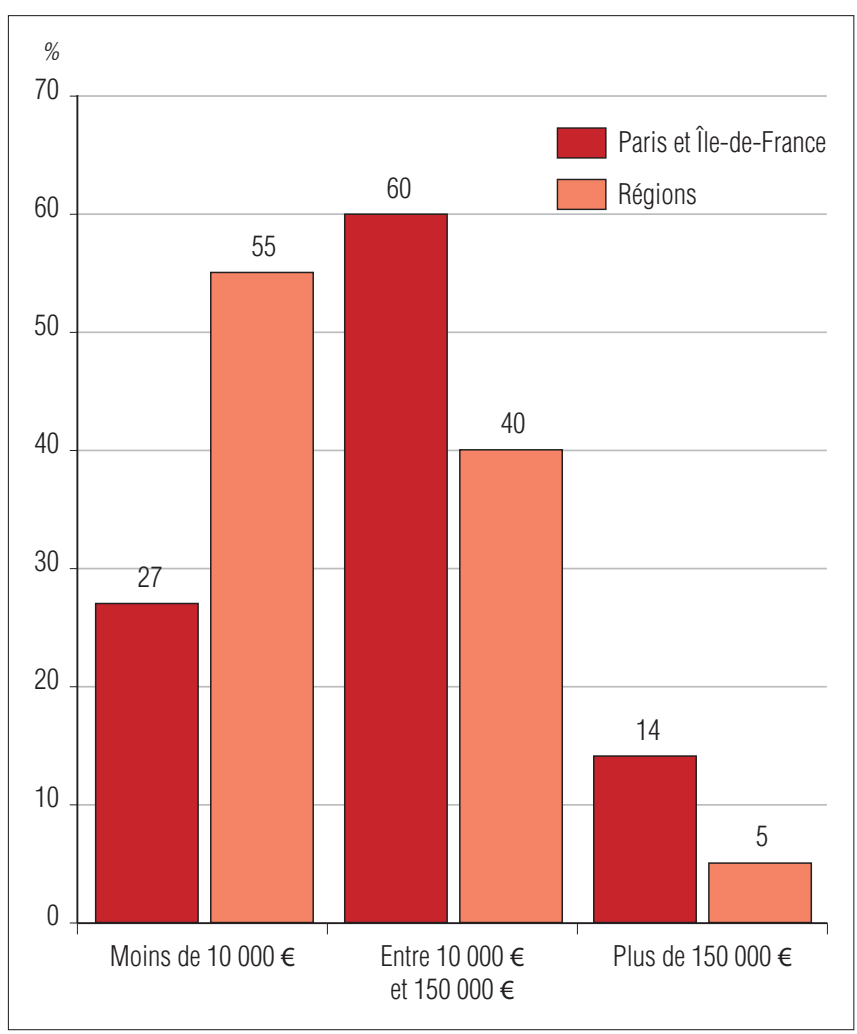

Source : DEPS, Ministère de la Culture et de la Communication, 2013.

Les inégalités de chiffre d'affaires apparaissent également fortes au regard de l'ancienneté des galeries. Les galeries d'art contemporain exerçant leur activité depuis plus de dix ans réalisent un chiffre d'affaires annuel moyen de 1,1 million d'euros, les deux tiers d'entre elles sont implantées à Paris ou en Île-de-France. De plus, en Île-deFrance, les galeries exerçant leur activité depuis plus de dix ans réalisent un chiffre d'affaires moyen plus de trois fois supérieur à celui des galeries plus jeunes. Cet écart n'est que de 1 à 1,5 en région (graphique 3 ).

On peut penser que les galeries plus anciennes se trouvent $a$ priori en position pour suivre leurs artistes qui, dans le déroulement de leur carrière, passent du premier au second marché et deviennent plus cotés et donc plus chers que lors de leur apparition sur le marché. Effectivement, ces deux facteurs forts de disparité entre galeries d'art contemporain que sont l'activité sur le second marché et l'ancienneté s'avèrent corrélés positivement. Plus l'ancienneté est élevée, plus l'intervention sur le second marché est fréquente : $25 \%$ pour les galeries de moins de 5 ans et de 5 à 10 ans, $28 \%$ pour celles de 10 à 20 ans et $32 \%$ pour celles de plus de 20 ans.

L'écart s'observe également dans les gammes de prix proposées par les galeries. Seulement $10 \%$ des galeries françaises proposent des œuvres d'art contemporain audelà du seuil des 150000 euros et, parmi elles, $82 \%$ sont implantées à Paris ou en Île-de-France.

Les niveaux de prix proposés par les galeries en région sont en moyenne plus faibles qu'en Île-de-France : $55 \%$ des galeries de région ne vendent pas d'œuvres de plus de 
10000 euros alors que $74 \%$ (les trois quarts) des galeries franciliennes le font (graphique 4). Cela s'explique vraisemblablement par le fait que les galeries en région sont celles qui proposent le plus d'artistes français, souvent moins cotés que les artistes internationaux.

\section{Volume d'affaires et présence à l'international}

La présence sur le marché international passe le plus souvent par la participation à des foires ou salons à l'étranger. Le fait de participer au moins à deux foires à l'étranger en 2011 introduit un clivage significatif entre galeries: celles n'ayant pas exposé à l'étranger en 2011 lors d'une foire ou salon et celles qui ne l'ont fait qu'une seule fois réalisent respectivement un chiffre d'affaires annuel moyen de 368000 euros et de 462000 euros. À l'opposé, les galeries présentes sur au moins deux foires ou salons à l'étranger - soit une présence internationale déjà affirmée - ont un chiffre d'affaires moyen de 3,3 millions d'euros, ce qui prouve l'importance de la demande internationale dans le volume global d'activité des galeries d'art contemporain (graphique 5).

Au total, plus de la moitié des galeries d'art contemporain françaises $(57 \%)$ déclarent avoir participé au moins à une foire (nationale ou internationale) en 2011. Le pourcentage de galeries participant à des foires en France (52\%) est, sans surprise, plus important que la part de celles représentées dans des foires et salons à l'étranger (31\%) (graphique 6).

Là encore, la fracture entre Paris et les régions est manifeste: la proportion de galeries participant aux foires et salons d'art contemporain (nationaux et internationaux) est nettement supérieure pour les galeries de Paris et d'île-deFrance $(68 \%)$ par rapport à celles établies en région (40\%).

$16 \%$ des galeries déclarent avoir plusieurs lieux d'expositions dans des villes différentes, en France ou à l'étranger. Ces galeries, minoritaires mais néanmoins importantes, présentent, sans surprise, des taux de participation aux foires et salons internationaux quatre fois supérieurs aux autres.

Les galeries présentes sur le marché international peuvent proposer des œuvres d'artistes contemporains de nationalité étrangère. Cette activité est en partie corrélée à la première au sens où participer à des foires à l'étranger n'est pratiquement le fait que de galeries présentant déjà des artistes étrangers ; néanmoins la moitié des galeries d'art contemporain présentant des œuvres d'artistes étrangers ne sont pas présentes dans des foires à l'étranger.

La présentation d'artistes étrangers est très fréquente puisqu'elle concerne près de quatre galeries sur cinq. Elle est, là encore, corrélée à un volume d'affaires élevé puisque le chiffre d'affaires moyen de ces galeries est de 930000 euros contre 190000 euros seulement pour les galeries proposant exclusivement des artistes français.

Cette corrélation entre volume d'affaires et présence sur le marché international peut se penser en termes de causalité : la demande est plus large dès lors qu'elle est internationale et, le marché international étant globalement plus important que le marché français, les niveaux de cotations y sont souvent plus élevés car les artistes contemporains étrangers sont plus valorisés que les Français. À l'inverse, les galeries proposant exclusivement des artistes français
Graphique 5 - Chiffre d'affaires moyen des galeries d'art contemporain selon la participation à des foires et salons à l'étranger en 2011

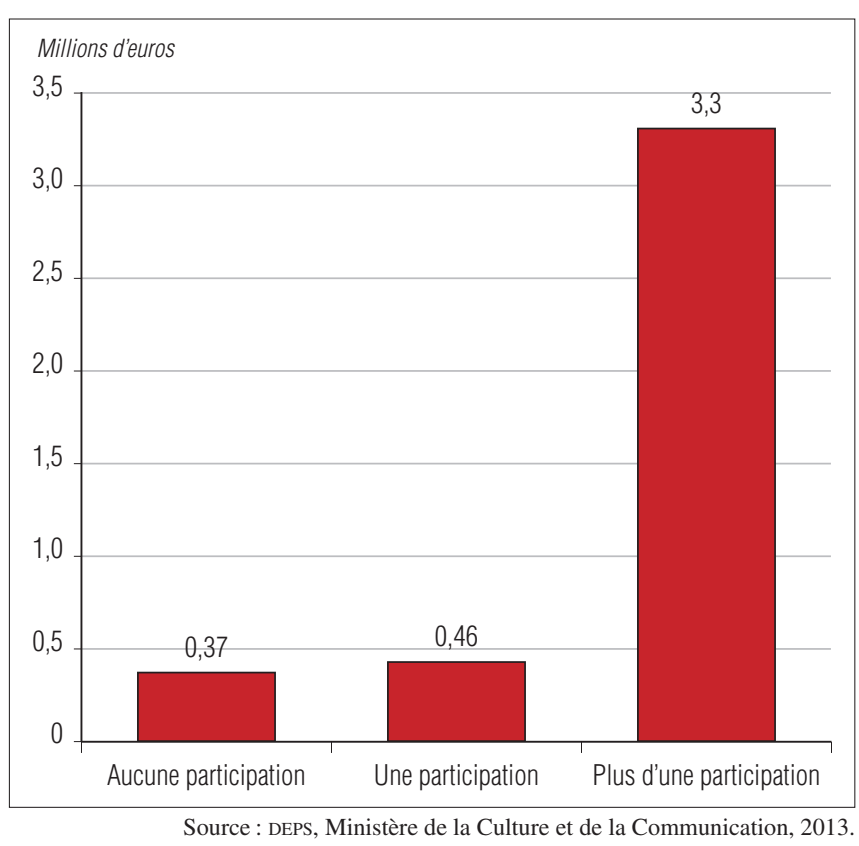

\section{Graphique 6-Répartition des galeries selon leur participation à au moins une foire d'art en 2011 et leur localisation}

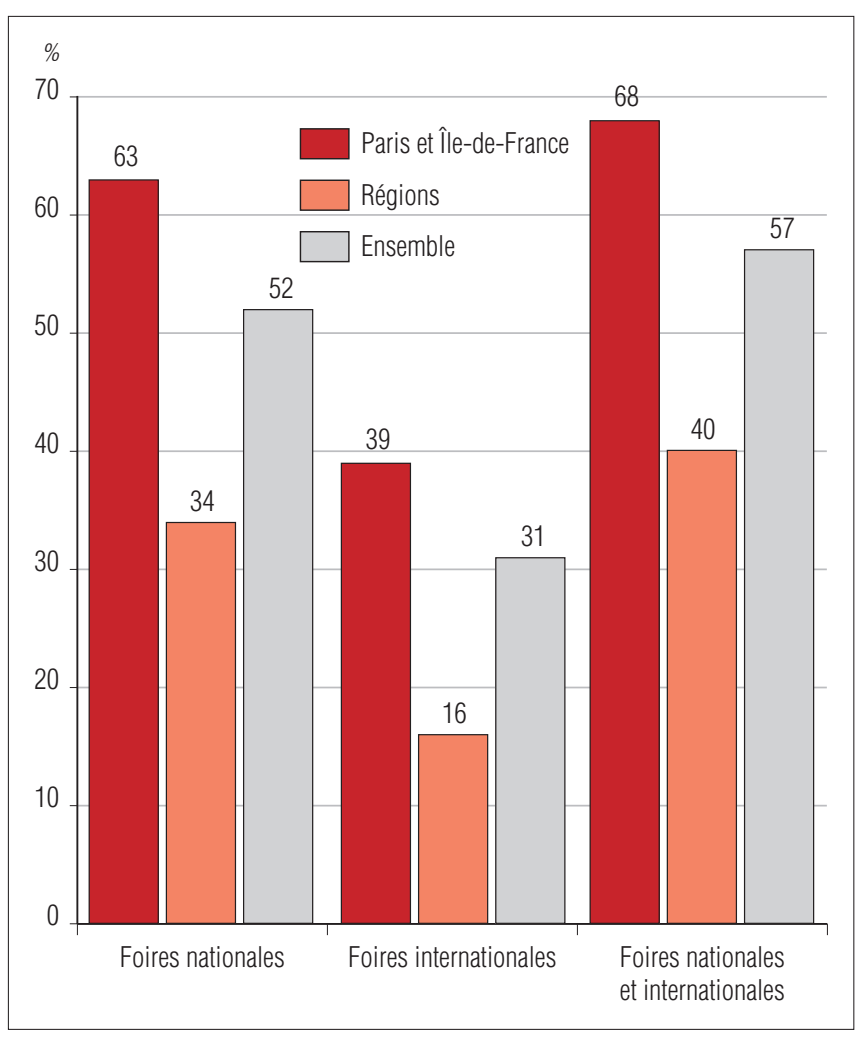

Source : DEPS, Ministère de la Culture et de la Communication, 2013. 
sont majoritairement des galeries de province, dont l'offre est très souvent composée d'artistes régionaux, moins cotés, de faible renommée, et conséquemment, moins chers.

On se gardera néanmoins de penser ces causalités de manière trop mécaniste car il existe des obstacles spécifiques à l'implantation internationale comme, par exemple, la difficulté de nouer des partenariats durables avec des galeries étrangères.

\section{Une activité largement tournée vers la promotion des artistes}

À rebours des facteurs forts de différenciation des galeries quant à leur volume d'affaires, les galeries d'art contemporain présentes sur le premier marché partagent des points communs importants.

C'est le cas, en premier lieu, de l'organisation d'expositions : quelle que soit leur taille, le rythme des expositions (individuelles ou collectives) des galeries est proche puisque $69 \%$ des galeries en ayant organisé en 2011 l'ont fait régulièrement à un rythme moyen allant d'une par trimestre à une par mois, soit entre 4 et 10 expositions au cours de l'année 2011. En moyenne, les galeries d'art contemporain françaises ont organisé un peu plus de cinq expositions au cours de l'année ${ }^{6}$. Il n'est observé, de plus, aucune corrélation significative entre le chiffre d'affaires annuel et le nombre d'expositions organisées au sein de la galerie.

Une galerie soutient en moyenne dix artistes français. $\mathrm{Ce}$ soutien est plus important au sein des galeries en région: $40 \%$ des galeries en région déclarent vendre les œuvres de plus de dix artistes français contre un quart (24\%) seulement des galeries de la capitale. De 2006 à 2008, les galeries d'art contemporain en région cotisant à la Maison des artistes présentaient en moyenne dix artistes français vivants contre sept seulement en moyenne pour les galeries parisiennes. Quant aux galeries enquêtées par CSA en 2005, elles travaillaient en moyenne avec une quinzaine d'artistes ou ayants droit vivant en France ou à l'étranger.

Une nette majorité de galeries d'art contemporain (62\%) ont accueilli la première exposition d'un artiste en 2011. Cette pratique est en partie liée à la focalisation sur le premier marché : $66 \%$ des galeries se consacrant au seul premier marché organisent de telles expositions alors que ce n'est le cas que de $54 \%$ de celles intervenant également sur le second marché.

Ce sont enfin $70 \%$ des galeries d'art contemporain qui déclarent contribuer directement au financement d'œuvres d'artistes qu'elles exposent. Cette pratique n'est pas liée à la présence ou non sur le second marché et également tout à fait indépendante du niveau d'activité et donc de la puissance financière de la galerie comme de la pratique de premières expositions. Il s'agit par ailleurs d'une pratique légèrement plus fréquente en région (36\% des galeries) qu'en Île-de-France (31\%).

Ainsi, finalement, une minorité (45\%) de galeries d'art contemporain se caractérise par la focalisation sur le premier marché et l'organisation de premières expositions. Le financement direct de la production d'œuvres n'y apparaît guère lié : cette pratique renvoie peut-être à la fois à un soutien matériel crucial à des artistes émergents mais aussi au nécessaire investissement dans une économie de la production à l'égard d'artistes plus reconnus.

\section{Un marché déjà largement internationalisé}

Une grande partie de l'activité des galeries françaises est tournée vers l'étranger, tant du point de vue de l'offre que de la demande, mais suivant des modalités très différentes.

Du point de vue de l'offre, $78 \%$ des galeries proposent des œuvres d'artistes de nationalité étrangère, tandis que du côté de la demande, près de la moitié de leur chiffre d'affaires total est réalisé à l'exportation : $44 \%$ de la part d'acheteurs particuliers étrangers, auxquels s'ajoutent les ventes à des institutions publiques étrangères (graphique 7). Au final, ce sont $87 \%$ des galeries d'art contemporain qui exportent - une proportion identique à celle de 1999 -, et $93 \%$ des galeries parisiennes et franciliennes.

Au-delà de cette présence généralisée sur le marché international - fût-ce de manière limitée et peu intensive car la présence à au moins une foire internationale ne concerne que $39 \%$ des galeries de Paris et d'île-de-France et $16 \%$ de celles de province -, une forte implication au niveau international reste un phénomène minoritaire. Ainsi, les seules galeries d'art contemporain qui réalisent plus de la moitié de leur chiffre d'affaires à l'exportation ne représentent que $15 \%$ de l'effectif national ${ }^{7} .80 \%$ d'entre elles sont parisiennes (contre $53 \%$ au total) et $42 \%$ d'entre elles sont actives sur le second marché (contre $28 \%$ ).

\section{Graphique 7 - Répartition du chiffre d'affaires des galeries d'art réalisé en France et à l'étranger en 2011}

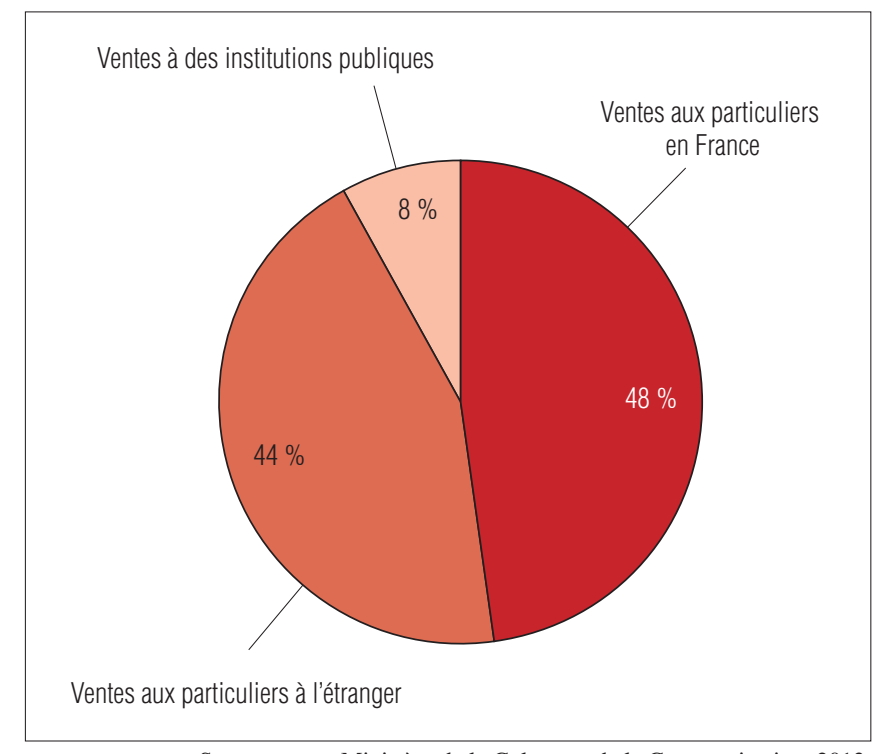

Source : DEPS, Ministère de la Culture et de la Communication, 2013.

6. L'enquête CSA de 2005 indiquait une moyenne de 8 expositions.

7. L'enquête de 1999 indiquait que plus du tiers des galeries répondantes (36\%) réalisaient la majorité de leur chiffre d'affaires à l'exportation, ce qui s'explique par une plus grande focalisation sur les galeries de promotion. 
Ces galeries ont un niveau d'activité particulièrement élevé et réalisent près du tiers du chiffre d'affaires total des galeries d'art contemporain alors qu'elles ne sont pas plus d'une sur six.

\section{UNE TYPOLOGIE DES GALERIES D'ART CONTEMPORAIN}

Afin de rendre compte de l'ensemble des spécificités des galeries d'art contemporain, de leur diversité et de leurs contrastes, deux approches complémentaires de segmentation statistique ont été menées.

\section{Deux directions majeures d'opposition suivant l'implantation géographique et l'ancienneté}

Les résultats de l'analyse des correspondances multiples $(\mathrm{ACM})^{8}$ portant sur les principales variables identifiant les caractéristiques de l'offre, du positionnement, et du mode de fonctionnement des galeries d'art contemporain, confirment des disparités particulièrement fortes dans deux directions déjà repérées : selon que les galeries sont franciliennes ou implantées en région, selon que les galeries sont jeunes (moins de dix ans d'existence) ou plus anciennes (plus de quinze ans).

Un premier axe oppose les galeries d'art contemporain franciliennes à celles situées en région: les premières se caractérisent principalement par un volume d'activité annuel de plus d'un million d'euros, et une forte participation aux foires. Au contraire, les galeries en région dégagent un volume d'activité annuel moyen de moins de $300000 €$ et sont, pour la plupart, absentes des foires et salons.

Un deuxième axe distingue les galeries selon leur ancienneté : les galeries exerçant leur activité depuis plus de quinze ans sont tournées vers le marché international, et ont une forte activité de soutien financier et de promotion de leurs artistes. Les galeries de moins de dix ans, en revanche, dégagent un volume d'activité annuel moyen plus faible (de $300000 €$ à un million d'euros).

\section{Une segmentation significative des galeries d'art contemporain en trois groupes ${ }^{9}$}

La classification ascendante hiérachique $(\mathrm{CAH})^{10}$ fait très nettement apparaître trois groupes relativement homogènes de galeries d'art contemporain en France : d'abord les galeries en région, majoritaires en nombre (classe 1) puis les galeries franciliennes, divisées en deux groupes d'importance égale: d'un côté, les galeries d'importance économique majeure (classe 3), de l'autre, des galeries plus secondaires de ce point de vue (classe 2). Le tableau suivant fournit les caractéristiques moyennes des galeries de chaque groupe.

Les galeries d'art contemporain situées en région constituent le groupe le plus important en nombre $(59 \%)$. Ce groupe se caractérise par la jeunesse des structures (généralement moins de dix ans d'existence), une activité principalement orientée sur le premier marché, une faible pré-

\section{Tableau 1 - Principales caractéristiques des galeries françaises d'art contemporain}

\begin{tabular}{|c|c|c|c|c|c|c|}
\hline $\begin{array}{l}\text { Implantation } \\
\text { à Paris- } \\
\text { Île-de-France }\end{array}$ & $\begin{array}{l}\text { Plusieurs lieux } \\
\text { d'exposition }\end{array}$ & $\begin{array}{l}\text { Forte intervention } \\
\text { sur le second } \\
\text { marché }\end{array}$ & $\begin{array}{l}\text { Présence } \\
\text { aux foires } \\
\text { et salons }\end{array}$ & $\begin{array}{l}\text { Prix maximal } \\
\text { des ventes d'œuvres } \\
\text { d'artistes vivants: } \\
150000 €\end{array}$ & $\begin{array}{l}\text { Chiffre d'affaires } \\
\text { supérieur à } \\
5 \text { millions d'€ }\end{array}$ & $\begin{array}{l}\text { CDI : > } 3 \text { salariés } \\
\text { Stagiaires : > à } 3 \\
\text { Temps complet : > à } 3\end{array}$ \\
\hline $\begin{array}{l}\text { Implantation } \\
\text { en région }\end{array}$ & Non significatif & Non significatif & $\begin{array}{l}\text { Absence } \\
\text { aux foires } \\
\text { et salons }\end{array}$ & $\begin{array}{l}\text { Prix maximal } \\
\text { des ventes d'œuvres } \\
\text { d'artistes vivants: } \\
10000 €\end{array}$ & $\begin{array}{l}\text { Chiffre d'affaires } \\
\text { inférieur à } \\
0,3 \text { millions d'€ }\end{array}$ & $\begin{array}{l}\text { CDI : aucun } \\
\text { Stagiaires : aucun } \\
\text { Temps complet : aucun }\end{array}$ \\
\hline
\end{tabular}

Axe 2 : principales caractéristiques selon l'ancienneté des galeries

\begin{tabular}{|c|c|c|c|c|c|}
\hline $\begin{array}{l}\text { Plus de } 15 \text { ans } \\
\text { d'existence }\end{array}$ & $\begin{array}{l}\text { Forte contribution } \\
\text { financière } \\
\text { à la production } \\
\text { d'œuvres }\end{array}$ & $\begin{array}{l}\text { Plus de } \\
10 \text { expositions } \\
\text { organisées } \\
\text { en } 2011\end{array}$ & $\begin{array}{l}\text { Peu ou pas } \\
\text { de lancement } \\
\text { d'artistes } \\
\text { émergents }\end{array}$ & $\begin{array}{l}\text { Activité tournée } \\
\text { vers le marché } \\
\text { international }\end{array}$ & $\begin{array}{l}\text { Chiffre d'affaires } \\
\text { entre } 1 \\
\text { et } 5 \text { millions d'€ }\end{array}$ \\
\hline $\begin{array}{l}\text { De } 0 \text { à } 10 \text { ans } \\
\text { d'existence }\end{array}$ & Non significatif & $\begin{array}{l}\text { Entre } 5 \\
\text { et } 10 \text { expositions } \\
\text { organisées } \\
\text { en } 2011\end{array}$ & $\begin{array}{l}\text { Lancement } \\
\text { d'artistes } \\
\text { émergents }\end{array}$ & $\begin{array}{l}\text { Activité tournée } \\
\text { vers le marché } \\
\text { national }\end{array}$ & $\begin{array}{l}\text { Chiffre d'affaires } \\
\text { entre } 0,3 \\
\text { et } 1 \text { million d'€ }\end{array}$ \\
\hline
\end{tabular}

8. L'analyse des correspondances multiples (ACM) est une méthode permettant le traitement de données individuelles issues de variables qualitatives. Elle offre une première description multidimensionnelle des données de l'enquête, et autorise la liaison des variables entre les galeries étudiées dans le but d'en dégager les principaux points de convergence ou de divergence.

9. En raison des données manquantes (notamment concernant le chiffre d'affaires), seules 176 des 225 galeries répondantes ont pu faire l'objet de cette classification qui a tiré parti des principaux axes factoriels issus de l'ACM précédente.

10. La classification ascendante hiérarchique ( $\mathrm{CAH})$ procède par fusions successives de groupes (clusters) déjà existants L'objectif de la CAH est de mettre en exergue une segmentation pertinente d'une population pour en faire ressortir les caractéristiques les plus marquantes. 


\begin{tabular}{|c|c|c|c|}
\hline & $\begin{array}{l}\text { Galeries } \\
\text { en région }\end{array}$ & $\begin{array}{l}\text { Galeries franciliennes } \\
\text { d'importance } \\
\text { secondaire }\end{array}$ & $\begin{array}{l}\text { Galeries franciliennes } \\
\text { d'importance } \\
\text { majeure }\end{array}$ \\
\hline $\begin{array}{l}\text { Pourcentage des } 176 \text { galeries } \\
\text { ayant répondu à } 100 \% \text { des questions } \\
\text { de l'enquête pilote }\end{array}$ & $59 \%$ & $20 \%$ & $20 \%$ \\
\hline \multicolumn{4}{|l|}{ Caractéristiques structurelles } \\
\hline Ancienneté moyenne & De 0 à moins de 10 ans & De 10 à 15 ans & plus de 15 ans \\
\hline Surface d'exposition moyenne & Moins de $100 \mathrm{~m}^{2}$ & De 100 à 200 m² $^{2}$ & Plus de $200 \mathrm{~m}^{2}$ \\
\hline Nombre moyen de salariés en CDI & 0,5 & 1,0 & 3,9 \\
\hline Nombre moyen de salariés en CDD & 0,1 & 0,1 & 0,6 \\
\hline Nombre moyen de stagiaires & 0,5 & 0,9 & 1,8 \\
\hline \multicolumn{4}{|l|}{ Positionnement } \\
\hline $\begin{array}{l}\text { à des foires nationales } \\
\text { Nombre moyen de participations }\end{array}$ & 0,7 & 1,2 & 1,7 \\
\hline à des foires internationales & 0,2 & 0,7 & 2,0 \\
\hline $\begin{array}{l}\text { Nombre moyen d'expositions réalisées } \\
\text { Nombre moyen d'artistes exposés }\end{array}$ & 4,7 & 5,8 & 6,6 \\
\hline pour la première fois & 2,9 & 2,1 & 2,3 \\
\hline Caractéristiques économiques & & & \\
\hline Chiffre d'affaires moyen & 0,2 million d'€ & 0,4 million $d^{\prime} €$ & 3 millions d'€ \\
\hline Chiffre d'affaires vente aux particuliers en France & 0,1 million $d^{\prime} €$ & 0,2 million d'€ & 1,5 million d'€ \\
\hline $\begin{array}{l}\text { Chiffre d'affaires vente aux particuliers à l'étranger } \\
\text { Prix de vente maximum }\end{array}$ & 0,07 million d'€ & 0,15 million d'€ & 1,8 million d'€ \\
\hline $\begin{array}{l}\text { des œuvres d'artistes vivants } \\
\text { Poids moyen des ventes d'artistes }\end{array}$ & Moins de $10000 €$ & 10000 à $150000 €$ & Plus de $150000 €$ \\
\hline $\begin{array}{l}\text { étrangers dans le chiffre d'affaires } \\
\text { Poids moyen des ventes aux particuliers }\end{array}$ & $18 \%$ & $33 \%$ & $37 \%$ \\
\hline étrangers dans le chiffre d'affaires & $21 \%$ & $34 \%$ & $39 \%$ \\
\hline
\end{tabular}

sence sur les foires et salons d'art contemporain, la vente d'œuvres d'artistes majoritairement français, des seuils de vente d'œuvres d'artistes vivants inférieurs à $10000 €$, un chiffre d'affaires annuel inférieur à $300000 €$.

Un deuxième groupe est constitué de galeries d'art franciliennes d'importance économique limitée (20\%). Ce groupe rassemble des galeries de dix à quinze ans d'existence, uniquement actives sur le premier marché, très présentes dans les foires et salons d'art contemporain, en France comme à l'étranger, apportant un important soutien aux artistes (lancement d'artistes émergents, soutien financier), réalisant un volume d'activité en deçà du seuil d'un million d'euros avec des prix de vente maximaux modestes pour les œuvres d'artistes vivants (entre $10000 €$ et $150000 €$ au maximum).

Enfin, le dernier groupe est constitué de galeries d'art contemporain franciliennes d'importance économique majeure $(20 \%)$, présentant comme principales caractéristiques d'exercer leur activité depuis plus de quinze ans, de disposer de plusieurs lieux d'expositions, d'intervenir fréquemment sur le second marché, d'avoir une activité de promotion et de communication très intense, semblable aux autres galeries franciliennes, de présenter une forte tendance à vendre des œuvres d'artistes étrangers, le prix de vente maximum étant souvent supérieur à $150000 €$ et, enfin, de réaliser un chiffre d'affaires annuel supérieur à un million d'euros.

\section{Graphique 8-Répartition des galeries d'art contemporain selon le type et l'ancienneté}

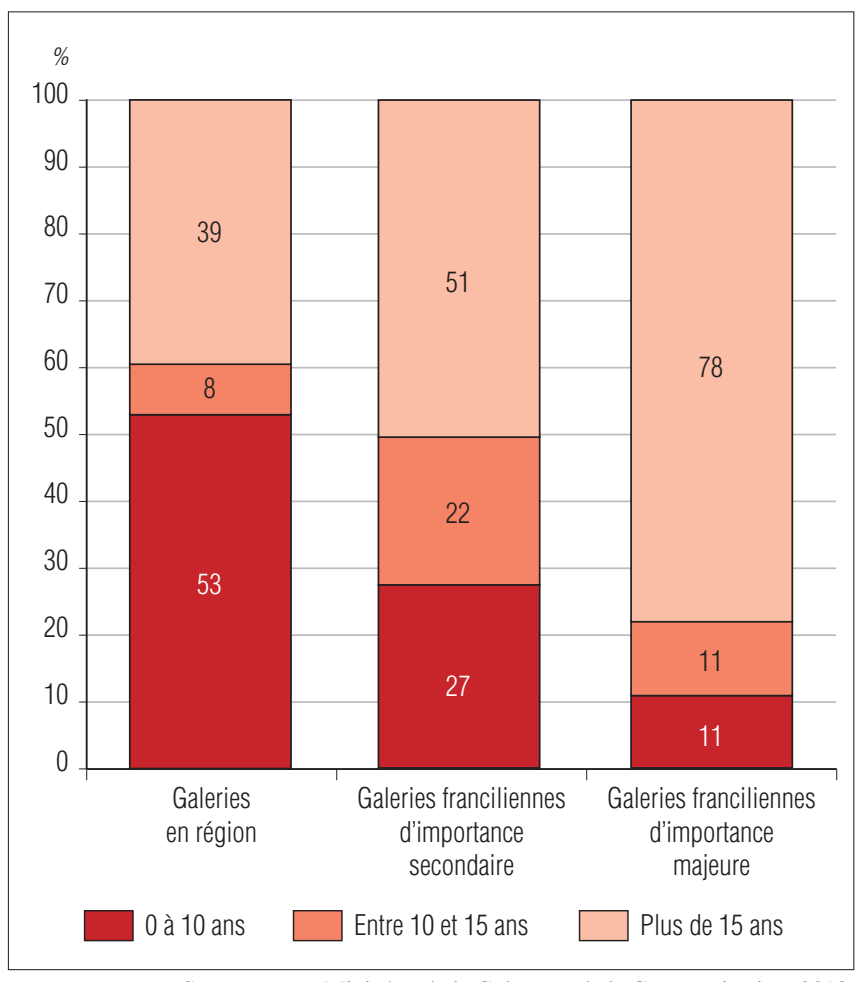

Source : DEPS, Ministère de la Culture et de la Communication, 2013. 


\section{Chiffre d'affaires et emplois}

\section{Une estimation du chiffre d'affaires de l'ordre d'un milliard d'euros}

Si l'on extrapolait les données recueillies au cours de l'enquête à l'ensemble des 2191 galeries de la base, on aboutirait à une estimation du chiffre d'affaires total réalisé en 2011 de 1,4 milliard d'euros, ce montant correspondant presque exclusivement à des ventes d'œuvres d'art (97\%), les autres recettes étant liées à des activités annexes (location d'espace, prestation d'encadrement, expertise...).

Cette estimation mérite néanmoins d'être relativisée pour deux raisons. D'abord la réalisation de l'enquête a montré le poids dans l'échantillon et, partant, au sein de la population, des cessations d'activité, des entreprises hors périmètre ou n'intervenant pas sur le premier marché, soit près de $20 \%$. Par ailleurs, les contacts non établis voire les refus peuvent correspondre pour partie respectivement à une absence d'activité ou une non-appartenance de fait au périmètre. Cela incite à penser que la population totale des galeries actives sur le premier marché serait peut-être inférieure de $25 \%$ à $30 \%$ à celle de départ (2 191), d'où une estimation du chiffre d'affaires total qui pourrait s'établir plutôt aux environs d'un milliard d'euros. Compte tenu, par ailleurs, de l'incertitude affectant cette estimation du fait de la taille de l'échantillon et de la disparité de taille entre galeries, l'estimation pour 2011 est plus élevée mais compatible avec celles disponibles précédemment (de l'ordre de 500 millions en 2006 d'après l'Insee pour environ 1500 galeries et 620 millions en 2011 d'après la Maison des artistes pour 1158 galeries $^{11}$ ).

Si une galerie sur trois estime, en septembre 2012, que sa situation économique va se dégrader, les galeries franciliennes sont significativement plus optimistes que les galeries en région : un quart des galeries d'art contemporain de la capitale envisage une amélioration de leur situation économique contre seulement $18 \%$ des galeries en région. Cette vision contrastée de l'avenir à court terme est certes liée aux incertitudes de la situation économique générale mais aussi aux difficultés et atouts propres à chaque galerie d'art contemporain prise individuellement ${ }^{12}$.

On note cependant que pour près de $40 \%$ des galeries interrogées, les cinq plus grands collectionneurs génèrent plus de $50 \%$ du chiffre d'affaires, ce qui induit une forte dépendance de la grande majorité des galeries à un nombre restreint de clients collectionneurs privés et peut être le signe d'une fragilité économique. Il s'agit d'un phénomène structurel qui semble se confirmer puisque ce pourcentage était déjà de $32 \%$ en 1999 pour les seules galeries d'art contemporain parisiennes.

\section{Rareté et précarité des emplois}

Les galeries d'art contemporain se caractérisent par la rareté et la précarité des emplois. Le nombre d'employés par galerie y est très faible, voire nul : près de la moitié des gale-

\section{Graphique 9 - Structure des effectifs employés}

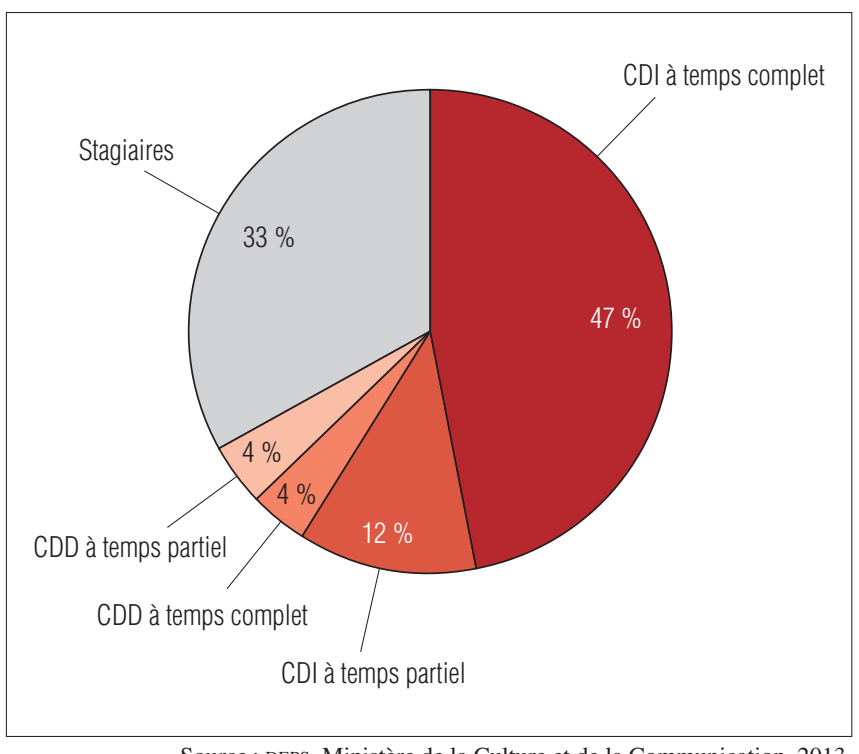

Source : DEPS, Ministère de la Culture et de la Communication, 2013.

ries d'art contemporain n'ont aucun salarié (47\%) - $49 \%$ en 1999 pour les galeries parisiennes -, et seules $5 \%$ des galeries emploient plus d'une personne. C'est ainsi que, au total, $54 \%$ des galeries n'emploient aucun salarié à durée indéterminée. Le CDI s'avère à cet égard plus fréquent en région qu'à Paris.

Les employés à temps complet représentent la plus grande partie de l'emploi salarié même si $78 \%$ des galeries d'art contem-porain n'ont rédigé aucun contrat à temps plein, un taux qui, à Paris et en Île-de-France, dépasse $82 \% .17 \%$ des galeries emploient un unique salarié à temps complet. Notons que la majorité des contrats salariés établis $(88 \%)$ sont cependant à durée indéterminée.

Au-delà de l'emploi salarié, en CDI ou en CDD, les contrats de stage concernent un tiers des emplois du secteur (36\% dans les galeries franciliennes, $27 \%$ en région). Ainsi, les CDD et les stages cumulés représentent $41 \%$ des effectifs des galeries, $43 \%$ dans les galeries à Paris et en Îlede-France et $38 \%$ au sein des galeries en région.

Extrapolés à l'ensemble des galeries d'art contemporain actives sur le premier marché, et avec les mêmes précautions que pour le chiffre d'affaires, les résultats de l'enquête permettent d'avancer un ordre de grandeur de 1800 contrats de travail, complétés par près d'un millier de stages.

\section{Quelles trajectoires pour les galeries d'art contemporain}

Cette recension et cette enquête portent sur un ensemble de galeries d'art contemporain plus important mais aussi plus hétérogène que celui souvent estimé à partir de la source de la Maison des artistes, ou retenu et étudié dans les enquêtes précédentes. Il faut à cet égard souligner la très grande diversité des postures artistiques et esthétiques. Pour

11. François RoUET, les Entreprises du commerce du marché de l'art, op. cit. et Chiffres clés pour la culture 2013, DEPS, La Documentation française, Paris, 2013.

12. L'enquête de CSA en 2005 laissait entendre que les responsables de galeries d'art contemporain répondants étaient sensiblement moins optimistes pour l'avenir du secteur que pour celui de leur galerie ou leur avenir professionnel personnel. 
autant, le dynamisme du secteur est une donnée structurelle manifeste au travers du nombre significatif d'artistes représentés, de la forte implication dans les foires et salons ainsi que dans la présence développée sur le marché international. Cela va de pair avec une disparité significative - sans être exceptionnelle - des chiffres d'affaires, mais surtout avec le poids de quelques très grandes galeries. Le chiffre d'affaires moyen est par ailleurs de 0,64 million d'euros contre 1,08 pour l'ensemble des secteurs marchands.

Quant au niveau d'emploi salarié, il est faible mais à relativiser sachant que les deux tiers des entreprises des secteurs marchands n'ont pas de salariés en 2011; on note cependant que le nombre moyen de salariés par entreprise est ici de 1,1 contre 2,2 dans les secteurs marchands culturels et 4,5 dans l'ensemble des secteurs marchands (hors agriculture). La particularité de l'emploi du secteur des galeries d'art contemporain tient plutôt à l'importance de l'emploi à temps partiel qu' au recours aux $\mathrm{CDD}$, légèrement inférieur à la moyenne nationale, et également au recours fréquent à des stagiaires.

Le chiffre d'affaires, variable d'une galerie à l'autre, est le signe à la fois de dynamiques de développement liées à l'investissement dans les foires et salons, de la montée en gamme des artistes présentés ou de la présence accrue sur le marché international, mais témoigne aussi de facteurs limitatifs comme la priorité aux artistes français voire l'implantation en région qui oblige à concilier périmètre d'action plus restreint et positionnement artistique souvent national.

Si la présente enquête vient d'abord conforter les résultats des travaux précédents en mettant l'accent sur le rôle de l'ancienneté des galeries, le faible emploi salarié ou encore la fragilité due à la dépendance à un petit nombre d'acheteurs, elle y ajoute la dimension géographique qui s'avère essentielle dans la définition de types.

Cette typologie atteste d'abord de l'importance quantitative des galeries implantées en région. Souvent jeunes et aux positionnements artistiques diversifiés ${ }^{13}$, ces structures disposent d'un périmètre d'action restreint et d'un positionnement artistique souvent national, voire régional. À côté, les galeries franciliennes - essentiellement parisiennes - se distinguent sur ce qui peut être perçu comme deux stades successifs de développement pour de petites galeries plutôt bien établies cherchant visiblement à se développer sur la scène internationale et à promouvoir toujours plus d'artistes, tout en affichant des gammes de prix limités et un chiffre d'affaires encore restreint. Leur perspective serait de développer volume d'activité et présence sur le marché international, en particulier en intervenant sur le second marché dans la revente de leurs artistes.

On peut y voir le prolongement de la typologie avancée dès l'étude de 1999 qui mettait en avant le cycle de vie d'une galerie d'avant-garde ${ }^{14}$. De même que l'on a pu mettre en avant l'existence de marchés successifs entre lesquels se déplacent les œuvres et les artistes ${ }^{15}$, on peut esquisser une trajectoire possible pour les galeries d'art contemporain qui consisterait à pouvoir passer - pas obligatoirement et plus ou moins vite - par trois périodes de développement correspondant aux trois types évoqués précédemment.

Dans une phase d'innovation d'environ dix années, la galerie débutante investira sur l'avenir en choisissant de soutenir un ou plusieurs artistes émergents, sans savoir s'ils seront porteurs ou non. Les prises de risques, les coûts de promotion et de communication sont importants dans cette étape de commencement: la galerie restreindra donc son champ d'action en limitant ses initiatives. Viendra ensuite une phase de croissance (de dix à quinze ans d'existence) : si la galerie a fait des choix stratégiques et artistiques judicieux, elle connaîtra une phase de croissance liée à la montée de la notoriété et de la cote des artistes promus. Elle s'ouvrira naturellement vers l'international, tant pour l'offre que pour la demande, mais en poursuivant ses efforts pour que ses artistes deviennent rentables. Enfin, après quinze ans d'existence, la galerie entrera dans une phase de rentabilité : les artistes soutenus aux débuts de l'existence de la galerie devenant une source de revenu. À ce stade, la rentabilité de ces derniers permettra à la galerie de promouvoir à nouveau de jeunes artistes. Cette trajectoire amène à s'interroger sur les conditions de création de nouvelles galeries à Paris et de développement des galeries en région.

13. L'investigation sur la diffusion et la valorisation de l'art actuel en région fournit une esquisse de ces questionnements : galeries de promotion, tremplin ou point de vente. Voir M. de Vrièse, B. Martin, C. Melin, N. Moureau, D. Sagot-Duvauroux, Diffusion et valorisation de l'art actuel en région..., op. cit.

14. F. Benhamou, N. Moureau, D. Sagot-Duvauroux, les Galeries d'art contemporain en France..., op. cit., p. 60.

15. Bertrand Rouget, Dominique Sagot-Duvauroux, Sylvie Pflieger, le Marché de l'art contemporain en France : prix et stratégies, Paris, Ministère de la Culture et de la Communicaiton, DEPS, la Documentation française, 1992, p. 105 sq. 


\section{Éléments de méthodologie}

La présente enquête a été réalisée en lien avec une investigation méthodologique sur les possibles enquêtes à mener pour mieux connaître l'activité des commerces de l'art dans leur ensemble. Dans cette perspective, un inventaire le plus exhaustif possible des sources disponibles a été réalisé ; ces dernières ont ensuite été expertisées quant à l'usage qui pouvait en être fait pour réaliser des enquêtes récurrentes. Ce travail a été réalisé par le cabinet Bluestone au cours du second semestre 2012.

Concernant les galeries d'art contemporain, ce travail de confrontation des différents fichiers a conduit à retenir 2191 unités économiques répertoriées comme galeries potentiellement actives sur le premier marché à partir des sources suivantes : Maison des artistes, Pages jaunes, Comité professionnel des galeries d'art, Artfacts.net, fichier de l'enquête csA de 2005. Cela a nécessité un dédoublonnage à la fois automatique et manuel. On a retenu comme caractérisation objective de l'activité sur le premier marché le négoce d'œuvres d'artistes en activité. Ce dernier est assimilé, par hypothèse, à la vente d'œuvres d'artistes vivants.

Un certain nombre d'ajustements conceptuels et sémantiques ont été nécessaires lors de la constitution de la base et de la préparation du questionnaire, notamment sur la distinction entre raison sociale et raison commerciale, la définition exacte des artistes français et étrangers, la définition respective de premier et second marché ou encore le périmètre exact de l'activité dite « hors France », compte tenu de la participation à des foires et salons à l'étranger.

L'enquête qui a porté sur la situation des galeries en septembre 2012 et leur activité de l'année 2011 a été finalement menée en deux vagues. Dans un premier temps, un échantillon représentatif de 301 galeries d'art contemporain a été constitué sur la base des deux seuls critères de représentativité disponibles sur une part suffisante de la base d'enquête constituée : l'implantation géographique au niveau du département et le niveau de notoriété établi par Artfacts. On a ainsi distingué deux catégo-

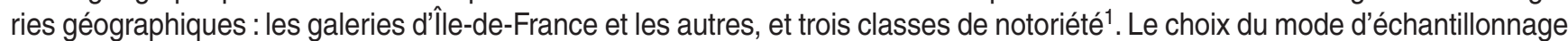
a été fait de manière à obtenir l'estimation la plus robuste possible du chiffre d'affaires total.

Ces galeries ont été contactées en septembre 2012 en même temps que le Comité professionnel des galeries d'art diffusait une information sur l'enquête auprès de l'ensemble de ses adhérents. Toutefois, au terme de la seconde semaine d'enquête, près d'un tiers des galeries de l'échantillon initial (98) ont dû être écartées : contacts non établis, structures hors périmètre, refus de répondre, cessation d'activité... L'échantillon initial a dû être enrichi de manière à obtenir un nombre suffisant de réponses et à garantir la fiabilité statistique des résultats et la robustesse des extrapolations : au total, l'échantillon initial a été porté à 428 galeries. 240 d'entre elles ont répondu au questionnaire. La question relative à la vente d'œuvres d'artistes vivants ayant révélé que 15 galeries interrogées n'étaient pas actives sur le premier marché, le nombre de réponses exploitables est finalement de 225. Le nombre de questionnaires renseignés systématiquement quant aux variables essentielles d'emploi et de chiffre d'affaires ainsi que sur un nombre suffisant d'autres variables était de 176, ce qui a permis d'intégrer les galeries correspondantes dans les analyses factorielles.

La représentativité des galeries répondantes permet d'extrapoler les résultats obtenus sur les différents thèmes abordés dans le questionnaire ainsi que d'avancer des ordres de grandeur pour le chiffre d'affaires et l'emploi total, en utilisant des pondérations adéquates. Le rapprochement avec les données de la Maison des artistes a permis d'estimer la structure régionale du chiffre d'affaires total. Enfin, on gardera à l'esprit que l'ensemble des données recueillies, en particulier sur le chiffre d'affaires, sont déclaratives.

1. Les galeries non retenues par Artfacts ont été regroupées dans une même catégorie. 


\section{RÉSUMÉ}

Les galeries d'art contemporain jouent un rôle essentiel dans l'émergence et la construction de la notoriété des artistes et, partant, dans la vitalité de la scène artistique française. Pourtant, l'analyse de leur activité est malaisée en l'absence d'une activité référencée dans la nomenclature d'activités française, ce qui oblige à croiser les sources. L'étude a opté pour une méthodologie inédite qui permet de dénombrer et caractériser les galeries d'art contemporain. Inégalement réparties sur le territoire, regroupées à Paris (48\% du total des galeries françaises), leur activité est significativement concentrée : $12 \%$ des galeries totalisent $72 \%$ du chiffre d'affaires annuel total. L'ancienneté de la galerie et sa présence sur le marché international sont des facteurs explicatifs importants de l'ampleur de son activité. Une typologie et une trajectoire type des galeries complètent l'analyse.

\section{ABSTRACT}

Contemporary art galleries play an essential role in the emergence and construction of artists' renown and, consequently, in the vitality of the French arts scene. However, in the absence of a benchmark activity in the French statistical classification of economic activities (NAF), it is hard to analyse, necessitating cross-referencing of sources. This study uses an original methodology which enables contemporary art galleries to be both counted and categorised. Distributed unevenly across the territory and clustered around Paris (containing 48\% of the total of French galleries), activity is highly concentrated: $12 \%$ of galleries account for $72 \%$ of their overall annual turnover. How long a gallery has been in existence and its status on the international market are important determining factors on the extent of its activity. The analysis concludes with a typical gallery trajectory and typology.

\section{Sur le même sujet :}

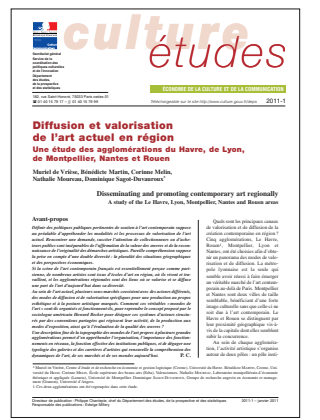

\section{Muriel de Vrièse, Bénédicte Martin, Corinne Melin, Nathalie Moureau, Dominique Sagot-Duvauroux Diffusion et valorisation de l'art actuel en région Une étude des agglomérations du Havre, de Lyon, de Montpellier, Nantes et Rouen}

Paris, DEPS, Ministère de la Culture et de la Communication, coll. «Culture études », 2011-1, janvier 2011

À partir de l'examen des différents canaux de diffusion et de valorisation de la création contemporaine dans les agglomérations de Lyon, Montpellier, Nantes, Le Havre et Rouen, l'étude propose une topographie des mondes de l'art actuel qui s'organise autour de pôles principaux: le monde institutionnel et le monde marchand, qui ont chacun leurs mondes intermédiaires. Elle identifie différents modèles économiques de l'art fondés sur une double distinction : économie d'œuvres versus économie de projet d'une part, innovation versus tradition d'autre part. L'étude propose une typologie inédite des galeries et met en évidence des profils d'artistes (formation, carrière, lieux de diffusion, rémunération) différents selon le modèle économique au sein duquel ces artistes évoluent.

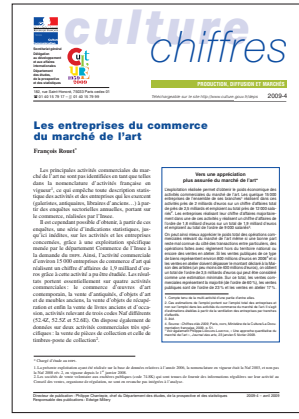

François Rouet

\section{Les entreprises du commerce du marché de l'art}

Paris, DEPS, Ministère de la Culture et de la Communication, coll. "Culture chiffres », 2009-4, avril 2009

L'exploitation d'indicateurs statistiques de quatre activités commerciales relevant de 3 codes NAF différents (52.4Z, $52.5 Z$ et $52.6 Z$ ) permet d'estimer le poids économique des activités commerciales du marché de l'art. [...]. Les entreprises réalisant leur chiffre d'affaires majoritairement dans une de ces activités y réalisent un chiffre d'affaires de l'ordre de 1,8 milliard d'euros sur un total de 1,9 milliard d'euros et emploient au total près de 9000 salariés. [...]

Tous les documents publiés par le DEPS sont téléchargeables sur http://www.culturecommunication.gouv.fr/Etudes-et-statistiques et sur www.cairn.info

Pour recevoir régulièrement les publications du DEPS et pour toute demande d'information: contact.deps@culture.gouv.fr 